\title{
Avoiding degradation of chemotherapy drugs: is graphene the answer?
}

\begin{abstract}
"While improving the target action of these drugs has been the center of an intense research effort, the potential for degradation by materials commonly used in their delivery, and the potential to create extremely harmful products appears to have been almost entirely overlooked."
\end{abstract}

\begin{abstract}
Keywords: catheter coatings $\bullet$ chemotherapy $\bullet$ photoemission spectroscopy $\bullet$ surface chemistry $\bullet$ synchrotron
\end{abstract}

\section{The reactivity of chemotherapy drugs}

Sadly, chemotherapy is an important part of many peoples' lives - approximately 850,000 people are undergoing treatment in the USA alone. The treatment typically involves the delivery of a cocktail of aggressive drugs into a patient's bloodstream, with the aim of inhibiting the development of cancer cells. Unfortunately, this drug cocktail often causes damage to healthy cells, including blood-forming cells in the bone marrow, hair follicles, cells in the mouth, digestive tract and reproductive system. Some drugs also cause damage to the heart, kidneys, bladder, lungs and nervous system [1]. In short, chemotherapy drugs are typically very reactive, and while they play an important part in cancer treatment, their reactivity with noncancerous cells is a major cause for concern. Exciting new targeted drug delivery methods are being proposed (see, for example, [2]), but these developments are still far from being put into general use.

Chemotherapy drugs are not just reactive toward cells, they are reactive agents more generally. This means that we should also be concerned by their interaction with all materials which they may encounter in normal use - for example, containers, needles, valves and the catheters through which the drugs are administered to the patient. Surprisingly, such concerns have been generally overlooked - few studies of the electronic properties of chemotherapy drugs [3] and the mechanics of catheter degradation have been carried out [4], but the chemical reactivity remains unknown. In our recent work, we have tried to address this by asking, do chemotherapy drugs damage delivery apparatus? Does the delivery apparatus damage the drugs? and can nanoscale coatings offer protection?

\section{Surface science methodology}

Over the last decades, the methodology for studying the chemistry of surfaces and interfaces has reached maturity. A wide range of experimental and computational techniques exist which allow the interactions of atoms, molecules and crystals to be observed in unprecedented detail - for example, to observe catalytic reactions in real-time, and to measure molecule orientation and bond conformation [5]. Experimental techniques such as x-ray photoelectron spectroscopy and scanning tunneling microscopy have been widely applied in many fields of research, as have computational methods, such as density functional theory. In fact these powerful methods are ideal for studying the chemistry and interactions of chemotherapy molecules with a wide range of materials, however, these methods have not been employed in understanding chemotherapy chemistry.

Studying reactive organic molecules, such as chemotherapy agents, using traditional surface science methodology is not entirely straightforward. The molecules may be
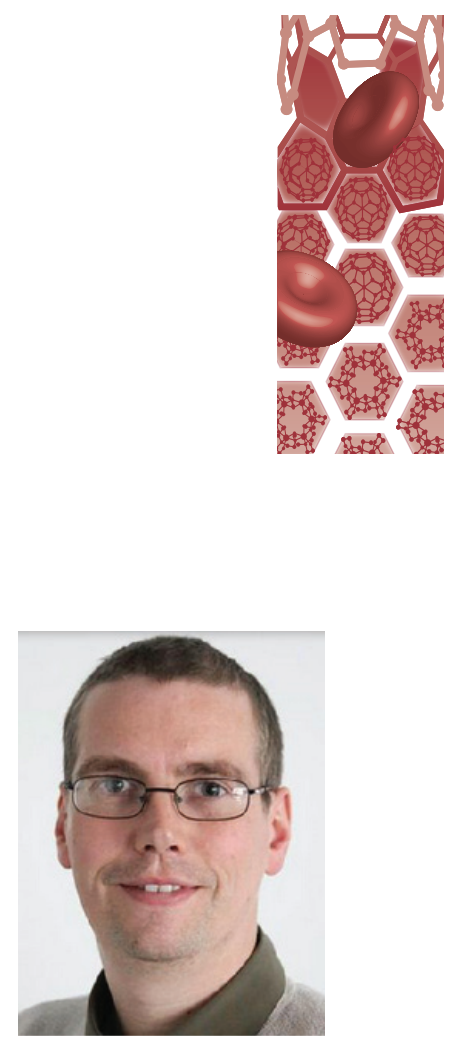

Justin W Wells

Department of Physics, Norwegian University of Science \& Technology (NTNU), NO-7491 Trondheim, Norway quantum.wells@gmail.com 
degraded by techniques involving exposure to $\mathrm{x}$-ray or electron beams, and their volatility can create problems for the ultra-high vacuum environment which surface science techniques typically require to operate. Even using a purely computational approach, large molecules such as epirubicin $\left(\mathrm{C}_{27} \mathrm{H}_{29} \mathrm{NO}_{11}\right)$ are sufficiently complex to make the computation demanding. Nonetheless, many of these potential obstacles are not unique to chemotherapy molecules and have been demonstrably circumvented for other organic molecules. Thus the exciting idea of applying the wealth of established surface science methods in this important and overlooked area is plausible.

\section{"Our initial studies are just the tip of the iceberg; we have been able to demonstrate that it is relatively straightforward to use surface chemistry techniques to gain a deep insight into the chemistry relevant to chemotherapy drug delivery."}

\section{Our research}

My research group at the Norwegian University of Science and Technology (NTNU) works in close collaboration with a number of internationally renown researcher groups on a wide variety of surface physics and $2 \mathrm{D}$ materials $[6-8]$. One of the favorite experimental methods is photoemission spectroscopy - which we perform in our own laboratory at NTNU or at synchrotron laboratories elsewhere in Scandinavia, and further afield. A natural starting point for us was to investigate the most common chemotherapy agents in our own laboratory, in order to test the feasibility of such a research venture. As part of a student summer project, we instigated in-vacuum studies of some of the most common breast cancer drugs; cyclophosphamide (a.k.a. Cytoxan), fluorouracil and epirubicin (a.k.a. Ellence). We initially studied the bulk properties of these drugs, and experimented with various means of sample preparation (i.e., preparing samples from powder, from solution and in-vacuum by thermal evaporation). Having demonstrated to ourselves that this methodology works, and that we were not inducing damage to the drugs, we moved the experiment to the Swedish synchrotron facility 'MAX-II' to make a more thorough investigation of the surface reactions relevant to the administering of chemotherapy.

Since these experiments are time consuming, and the synchrotron time is limited, we focused on one particular question; silver is used increasingly as a coating in medical applications in general, and more recently in catheters for chemotherapy [9-12], is it possible that silver coatings are reacting with halogenated drug molecules, such as fluorouracil? Indeed, we found out that silver catalytically breaks the C-F bond in the fluorouracil molecule and facilitates a release of strongly acidic HF [13]. Our study primarily uses photoemission to observe this, and this is complemented by Auger spectroscopy, x-ray absorption spectroscopy, scanning tunneling microscopy and density functional theory calculations. There are several important implications of this finding; silver is degrading the drugs and potentially compromising the efficacy of a treatment, but the accidental release of HF may be responsible for catheter degradation and the associated medical complications [4]. The possibility of releasing HF into an already sick patient's bloodstream is also a serious concern [14].

\section{Graphene?}

Having identified that silver catalytically degrades fluorouracil, the obvious question to ask is can we find a material which does not degrade these drugs? There are a large number of potential candidates, and we anticipate that we will conduct a more systematic survey in the future, but in our initial study one material stood out as being especially relevant; graphene. Graphene has been hailed recently as a wonder nanomaterial - it is strong, light, thin, cheap/plentiful and most importantly for us, inert and biocompatible. While graphene has garnered much interest as a 'single atom thick' protective coating $[15,16]$ and has been proposed as an ideal candidate for biocompatible coatings [17], graphene's potential in medicine remains generally unexplored (for example, a search of Nature Medicine returns 0 articles [18]). In addition to these considerations, my research group has experience with preparing and studying high quality graphene for other research projects [19-21] and hence we took graphene to be our prime candidate for this study.

We conducted a surface chemistry experiment which was a close analog to the aforementioned experiment with silver - but in this case we investigated the interface chemistry between fluorouracil and graphene. As expected, we found that the defluorination which was prevalent on silver was completely absent on the graphene surface. In fact, the interaction between the chemotherapy molecules and the graphene is so weak that the molecules do not even stick to it unless the temperature is far below body temperature. In short, graphene offers an inert and 'nonstick' surface which would make it an ideal candidate for coating catheters and the apparatus used in the delivery of fluorouracil.

\section{Outlook}

Our initial studies are just the tip of the iceberg; we have been able to demonstrate that it is relatively straightforward to use surface chemistry techniques to gain a deep insight into the chemistry relevant to chemotherapy 
drug delivery. We have also shown that these studies are of great importance since chemotherapy drugs can be easily degraded by common materials - and we have shown that this can be avoided by choosing emerging new materials as protective coatings.

On the other hand, there is much work to be done. Our studies so far are limited to silver and graphene. Furthermore, they are limited to pristine/idealistic surfaces which do not accurately reproduce the patient/ drug environment. Does a graphene coating still perform well if it gets damaged? Is it possible to make high quality graphene coatings on the inside of flexible catheters? There are many such questions left to be answered.

Our initial investigations have focused on the halogenated drug fluorouracil, since we suspected that a dehalogenation reaction could occur - are the other common chemotherapy drugs just as readily degraded? What about the plethora of emergent and targeted chemotherapy agents which are coming to the market? Although there are many questions yet to be answered, we have presented the methodology available to answer them. At NTNU, we now have two master students working on some of these questions. We have also applied for funding from the Norwegian Research Council to give us the resources to facilitate further studies.

\section{What do we learn from this?}

The most important thing we learn here is that chemotherapy drugs are reactive molecules, and therefore, we need to give due consideration to the materials

\section{References}

1 American Cancer Society. www.cancer.org

2 Liu Y, Miyoshi H, Nakamura M. Nanomedicine for drug delivery and imaging: a promising avenue for cancer therapy and diagnosis using targeted functional nanoparticles. Int. J. Cancer 120, 2527-37 (2007).

3 de Aguiar HB, Cunha FGC, Nart FC, Miranda PB. Adsorption behavior of 5-fluorouracil on $\mathrm{Au}(111)$ : an in situ STM study. J. Phys. Chem. C 114, 6663-6670 (2010).

4 Ardalan A, Flores M. A new complication of chemotherapy administered via permanent indwelling central venous catheter. Cancer 75, 2165-8 (1995).

5 Somorjai GA, Li Y. Impact of surface chemistry. Proc. Natl Acad. Sci. USA 108, 917 (2011).

6 Wells JW, Handrup K, Kallehauge JF et al. The conductivity of $\mathrm{Bi}(111)$ investigated with nanoscale four point probes. J. Appl. Phys. 104, 5, 053717 (2008).

7 Riley JM, Mazzola F, Dendzik M et al. Direct observation of spin-polarized bulk bands in an inversion-symmetric semiconductor. Nat. Phys. 10, 11, 835-839 (2014). which they come into contact with. While improving the target action of these drugs has been the center of an intense research effort, the potential for degradation by materials commonly used in their delivery, and the potential to create extremely harmful products appears to have been almost entirely overlooked. Demonstrating that developed surface science methods can be applied to this problem should facilitate a rapid improvement in knowledge in this area and we hope that we will soon see an improved choice of materials and nanoscale coatings in use in the near future.

\section{Acknowledgements}

The MAX-IV laboratory, and in particular A Preobrajenski of beamline D1011, AA Cafolla (Dublin) performed scanning tunneling microscopy measurements for us, T Trinh (under the supervision of S Kjelstrup) performed the density functional theory calculations using the NOTUR national computing infrastructure. The measurements were performed by students and postdoctoral researchers in my group and collaborating groups (F Mazzola, E Østli, S Cooil, ETB Skjønsfjell and K Høydalsvik) and supervised by DA Evans and DW Breiby.

\section{Financial \& competing interests disclosure}

The author has no relevant affiliations or financialinvolvement with any organization or entity with a financial interest in or financial conflict with the subject matter or materials discussed in the manuscript. This includes employment, consultancies, honoraria, stock ownership or options, expert testimony, grants or patents received or pending, or royalties.

No writing assistance was utilized in the production of this manuscript.

8 Miwa JA, Hofmann P, Simmons MY, Wells JW. Direct measurement of the band structure of a buried twodimensional electron gas. Phys. Rev. Lett. 110, 136801 (2013).

9 Timsit JF, Dubois Y, Minet C et al. New materials and devices for preventing catheter-related infections. Ann. Intensive Care 1, 1 (2011).

10 Armentano I, Arciola CR, Fortunati E et al. The interaction of bacteria with engineered nanostructured polymeric materials: a review. Sci. World J. 201418 (2014).

11 Suska F, Svensson S, Johansson A et al. In vivo evaluation of noble metal coatings. J. Biomed. Mater. Res. B 92B, 86-94. (2010).

12 Harter C, Salwender H, Bach A, Egere G, Goldschmidt $\mathrm{H}, \mathrm{Ho}$ A. Catheter-related infection and thrombosis of the internal jugular vein in hematologic-oncologic patients undergoing chemotherapy: a prospective comparison of silver-coated and uncoated catheters. Cancer 94, 245-51 (2002).

13 Mazzola F, Trinh T, Cooil S. Graphene coatings for chemotherapy: avoiding silver-mediated degradation. 2D Materials 2, 025004 (2015). 
14 Yamashita M, Suzuki M, Hirai H, Kajigaya H. Iontophoretic delivery of calcium for experimental hydrofluoric acid burns. Crit. Care Med. 29, 1575-8 (2001).

15 Nilsson L, Andersen M, Balog R et al. Graphene coatings: probing the limits of the one atom thick protection layer. ACS Nano 6, 10258-10266 (2012).

16 Kirkland NT, Schiller T, Medhekar N, Birbilis N. Exploring graphene as a corrosion protection barrier. Corros. Sci. 56, $1-4$ (2012).

17 Zhang W, Lee S, McNear K et al. Use of graphene as protection film in biological environments. Sci. Rep. doi:10.1038/srep04097 (2014) (Epub ahead of print).
18 Dolgin E. Nature medicine spoonful of medicine odds and ends.

http://blogs.nature.com

19 Mazzola F, Wells JW, Yakimova R et al. Kinks in the sigma band of graphene induced by electron-phonon coupling. Phys. Rev. Lett. 111, 216806 (2013).

20 Vinogradov NA, Simonov KA, Zakharov AA et al. Hole doping of graphene supported on $\operatorname{Ir}(111)$ by AlBr3. Appl. Phys. Lett. 102, 061601 (2013).

21 Perkins E, Barreto L, Wells J, Hofmann P. Surface-sensitive conductivity measurement using a micro multi-point probe approach. Rev. Sci. Instrum. 84, 3, 033901 (2013). 